Modern Physics Letters A,

(C) World Scientific Publishing Company

\title{
BALMER-LIKE SERIES FOR BARYON RESONANCES
}

\author{
M. LOSANOW-KIRCHBACH * \\ Institut für Kernphysik, J. Gutenberg Universität, D-55099 Mainz, Germany
}

In memory of my first teacher on group theory, the Bulgarian physicist

Angel Nicolov.

\begin{abstract}
The pole positions of various baryon resonances have been found to reveal a well pronounced clustering, the so-called Höhler cluster. In a previous work, the Höhler clusters have been shown to be identical to Lorentz multiplets of the type $\left\{\frac{1}{2}+l^{\prime}, \frac{1}{2}+l^{\prime}\right\} \otimes$ $\left[\left\{\frac{1}{2}, 0\right\} \oplus\left\{0, \frac{1}{2}, 0\right\}\right]$ with $l^{\prime}$ integer. Here we show that the cluster positions are well described by means of a Balmer-series like recursive mass formula.
\end{abstract}

One of the oldest unsolved problems of baryon spectroscopy is the relativistic description of resonances with higher-spin. This problem appears both in treating the higher-spin states as pointlike objects as well as at the composite particle level in the various quark models. In the first case the problem is caused by the presence of redunant components, that need be eliminated, in the multi-spinor representations of the Lorentz group embedding the higher-spin states considered. In the second case, the problem is mainly due to the improper choice for the symmetry group of the constituent quark model.

As known, baryons have been considered so far to constitute multiplets of the group $\mathrm{O}(3) \otimes \mathrm{SU}(6)_{S F}$. Through this group the trivial spin-flavor $(S F)$ correlation between three quarks in the 1s-shell is naively extended to arbitrary orbital angular momenta. In this way the relativistic description of the higher-spin states is completely spoiled. Indeed, the Lorentzian boost breaks up the spin-flavor correlation. It couples the spin to different $\mathrm{O}(3)$ representations and produces mixing between the $\mathrm{O}(3) \otimes \mathrm{SU}(6)_{S F}$ multiplets. The problems raised by symmetry groups of strong interaction based on strong correlations between the spin- and flavor degrees of freedom of quarks don't have only conceptual disadvantages. Also their predictions are not satisfactory. For example, the $\mathrm{O}(3) \otimes \mathrm{SU}(6)_{S F}$ classification scheme predicts numerous still unobserved higher-spin states known as 'missing resonances'. Finally, the spacing among the corresponding multiplets is much smaller as compared to the mass splitting between the multiplet members. On the other side, a recent analysis of the pole positions of various baryon resonances $\left(L_{2 I, 2 J}\right)$ with masses below $\sim 2500 \mathrm{MeV}$ performed by Höhler et al. [1] reveals a well-pronounced clustering. This is quite a surprising result in as it was not anticipated by any model or theory 2. In the following, these baryon clusters will be referred to as 'Höhler clusters' according to a suggestion of Nefkens [2]. In conjunction with this observation, the symmetry of all reported $N, \Delta$ and $\Lambda$ baryon excitations with masses below 2500

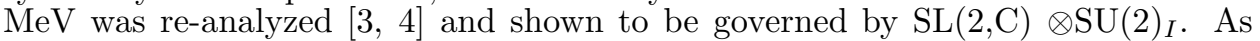
long as the group $\mathrm{SL}(2, \mathrm{C})$ is the universal covering of the Lorentz group, the new classification scheme for baryons is determined by $\mathrm{O}(1,3) \otimes \mathrm{SU}(2)_{I}$ rather than by $\mathrm{O}(3) \otimes \mathrm{SU}(6)_{S F}$ as always used since the invention of the naive three flavor quark model. The $\mathrm{O}(1,3) \otimes \mathrm{SU}(2)_{I}$ symmetry indicates that the spin-orbital correlation

*E-mail: mariana@kph.uni-mainz.de 
between quarks is much stronger than the spin-flavor one. Indeed, in Refs. 3, \#, it was demonstrated that Höhler's clusters are identical to Lorentz multiplets of the type $\left\{\frac{1}{2}+l^{\prime}, \frac{1}{2}+l^{\prime}\right\} \otimes[\{1 / 2,0\} \oplus\{0,1 / 2\}]$ with $l^{\prime}$ integer. These representations can be interpreted to decribe a quark coupled to Di-quarks of (integer) spins $l=0, \ldots, 2 l^{\prime}+1$ as emerging through the coupling of two spin- $1 / 2$ objects occupying an $l^{\prime}$ shell. Indeed, the covariant quark-Di-quark model based on solving the Bethe-Salpeter equation reveals an internal $\mathrm{O}(4)$ symmetry as visible from the rapid convergence of its solutions in the basis of the Gegenbauer polynomials, the orthogonal polynomials of the group $\mathrm{O}(4)$, an observation reported in Refs. [5].

The Lorentz multiplets introduced above are well known from the Coulomb problem, where they correspond to even principal quantum numbers $n=2 l^{\prime}+2$. In the following, these multiplets will be sometimes termed as Coulomb-spinors. All orbital angular momenta contained within a Coulomb multiplet have either natural or unnatural parity. Coupling a Dirac spinor to $l$ is then standard and leads to states with total spins $\vec{J}=\vec{l} \otimes \frac{\overrightarrow{1}}{2}$ with $l=0, \ldots, 2 l^{\prime}+1$. The parity $(-1)^{L+1}$ of a single $\pi N$ resonance $L_{2 I, 2 J}$ in standard notation $\theta$ is determined in the present classification scheme by either $(-1)^{l}$ or $(-1)^{l+1}$, depending on whether the parity of the intrinsic orbital angular momentum is natural or unnatural. In the present notation, $\mathrm{L}$ takes the values of either $L=|l-1|,(l+1)$ for natural, or $L=l$ for unnatural parities.

The new baryon spectrum generating algebra $\mathrm{o}(1,3) \otimes \mathrm{su}(2)_{I}$ has numerous advantages over the old $\mathrm{o}(3) \otimes \mathrm{su}(6)_{S F}$ one. For example, the spacing of about 200 $\mathrm{MeV}$ among the relativistic multiplets with masses below $2000 \mathrm{MeV}$ becomes much larger as compared to the mass splitting of 50-70 MeV between the corresponding multiplet members. Furthermore, fewer 'missing resonances' are predicted. For example, within the $\mathrm{O}(1,3) \otimes \mathrm{SU}(2)_{I}$ scheme, the $\Delta$ spectrum below $2 \mathrm{GeV}$ appears complete. As the $\mathrm{F}_{37}$ state has to be paralleled in the nucleon sector by a (still unobserved) $\mathrm{F}_{17}$ resonance with a mass around $1700 \mathrm{MeV}$, only that latter state has to be viewed as a 'missing resonance' among the non-strange baryon excitations with masses below $2000 \mathrm{MeV}$. In comparing the states from the third nucleon and $\Delta$ clusters, four more missing resonances are predicted. These are the $\mathrm{H}_{1,11}, \mathrm{P}_{31}$, $\mathrm{P}_{33}$, and $\mathrm{D}_{33}$ states with masses between 2200 and $2400 \mathrm{MeV}$.

In summary, five new, still unobserved non-strange resonances have been predicted in Refs. 3, 4t (see Table 1).

The major advantage of the relativistic spectrum generating algebra for baryons is, however, that it reconciles such seemingly contrary ideas of the baryon structure like the constituent quark model on the one side, and the structureless (pointlike) view of hadrons, on the other side. Indeed, the Lorentz representations used for describing pointlike higher-spin states emerge in a natural way from an underlying quark-Di-quark picture of baryon structure. In that case the lower-spin components of the Lorentzian multi-spinors embedding the higher-spin states are not any longer redundant but correspond to observed resonances. As a consequence, the relativistic description of higher-spin states becomes possible because the relativistic propagators of the Höhler clusters can be directly read off from the representation theory of the Lorentz group. For example, the $\left(S_{2 I, 1}, D_{2 I, 3}\right)$ cluster is described in terms of a Lorentz vector with Dirac spinor components and its propagator is given by (see Ref. [4 for details )

$$
S_{\mu \nu}=\frac{\left(p^{\lambda} \gamma_{\lambda}+M\right)\left(g_{\mu \nu}-\frac{p_{\mu} p_{\nu}}{M^{2}}\right)}{2 M\left(p^{2}-M^{2}\right)}, \quad \mu=0,1,2,3,
$$

with $\mathrm{M}$ standing for the mass of the degenerate resonances under considerations. In noting that, say, the first $\mathrm{S}_{11}$ and $\mathrm{D}_{13}$ states appear separated by only $15 \mathrm{MeV}$, one sees that the relativistic contribution of these states to the amplitude of processes 
Table 1. Correspondence between Höhler clusters and Lorentz multiplets. The five predicted missing resonances here have been labeled by ' $m s$ '.

\begin{tabular}{|c|c|c|}
\hline $\mathbf{L}_{2 \mathrm{I}, 2 \mathrm{~J}}$ States & Pole $(\mathrm{MeV})$ & Lorentz Multiplet \\
\hline $\begin{array}{l}\mathrm{S}_{11}, \mathrm{P}_{11}, \mathrm{P}_{13}, \mathrm{D}_{13} \\
\mathrm{D}_{15}, \mathrm{~F}_{15}, \mathrm{~F}_{17}^{m s}\end{array}$ & $\begin{array}{l}(1665 \pm 25) \\
-(55 \pm 15) \mathrm{i}\end{array}$ & $\begin{array}{l}\left\{\frac{3}{2}, \frac{3}{2}\right\} \otimes\left[\left\{\frac{1}{2}, 0\right\} \oplus\left\{0, \frac{1}{2}\right\}\right] \\
\otimes\left\{\frac{1}{2}\right\}_{I} \\
(\mathrm{n}=4)\end{array}$ \\
\hline $\begin{array}{l}\mathrm{S}_{11}, \mathrm{P}_{11}, \mathrm{P}_{13}, \mathrm{D}_{13}, \\
\mathrm{D}_{15}, \mathrm{~F}_{15}, \mathrm{~F}_{17}, \mathrm{G}_{17}, \\
\mathrm{G}_{19}, \mathrm{H}_{19}, \mathrm{H}_{1,11}^{m s}\end{array}$ & $\begin{array}{l}(2110 \pm 50) \\
-(180 \pm 50) \mathrm{i}\end{array}$ & $\begin{array}{l}\left\{\frac{5}{2}, \frac{5}{2}\right\} \otimes\left[\left\{\frac{1}{2}, 0\right\} \oplus\left\{0, \frac{1}{2}\right\}\right] \\
\otimes\left\{\frac{1}{2}\right\}_{I} \\
(\mathrm{n}=6)\end{array}$ \\
\hline $\begin{array}{l}\mathrm{S}_{31}, \mathrm{P}_{31}, \mathrm{P}_{33}, \mathrm{D}_{33} \\
\mathrm{D}_{35}, \mathrm{~F}_{35}, \mathrm{~F}_{37}\end{array}$ & $\begin{array}{l}(1820 \pm 30) \\
-(120 \pm 30) \mathrm{i}\end{array}$ & $\begin{array}{l}\left\{\frac{3}{2}, \frac{3}{2}\right\} \otimes\left[\left\{\frac{1}{2}, 0\right\} \oplus\left\{0, \frac{1}{2}\right\}\right] \\
\otimes\left\{\frac{3}{2}\right\}_{I} \\
(\mathrm{n}=4)\end{array}$ \\
\hline $\begin{array}{l}\mathrm{S}_{31}, \mathrm{P}_{31}^{m s}, \mathrm{P}_{33}^{m s}, \mathrm{D}_{33}^{m s}, \\
\mathrm{D}_{35}, \mathrm{~F}_{35}, \mathrm{~F}_{37}, \mathrm{G}_{37} \\
\mathrm{G}_{39}, \mathrm{H}_{39}, \mathrm{H}_{3,11}\end{array}$ & less established & $\begin{array}{l}\left\{\frac{5}{2}, \frac{5}{2}\right\} \otimes\left[\left\{\frac{1}{2}, 0\right\} \oplus\left\{0, \frac{1}{2}\right\}\right] \\
\otimes\left\{\frac{3}{2}\right\}_{I} \\
(\mathrm{n}=6)\end{array}$ \\
\hline $\begin{array}{l}\mathrm{S}_{01}(1800), \mathrm{P}_{01}(1810) \\
\mathrm{D}_{05}(1830), \mathrm{F}_{05}(1820) \\
\mathrm{P}_{03}(1890), \mathrm{D}_{03}^{m s}(2000 ?) \\
\mathrm{G}_{07}(2100), \mathrm{F}_{07}(2020)\end{array}$ & & $\begin{array}{l}\left\{0^{ \pm}\right\} \otimes\left[\left\{\frac{1}{2}, 0\right\} \oplus\left\{0, \frac{1}{2}\right\}\right] \\
\left\{0^{ \pm}\right\} \otimes\left[\left\{\frac{5}{2}, 0\right\} \oplus\left\{0, \frac{5}{2}\right\}\right] \\
\left\{0^{ \pm}\right\} \otimes\left[\left\{\frac{3}{2}, 0\right\} \oplus\left\{0, \frac{3}{2}\right\}\right] \\
\left\{0^{ \pm}\right\} \otimes\left[\left\{\frac{7}{2}, 0\right\} \oplus\left\{0, \frac{7}{2}\right\}\right]\end{array}$ \\
\hline
\end{tabular}


M. Losanow-Kirchbach

like meson photoproduction at threshold, can easily be calculated. Along the line of the representation theory of the Lorentz group, the construction of higher-cluster propagators is straightforward.

Table 2. Predicted $\left(M_{j j}^{t h}\right)$ and reported $\left(M_{j j}^{e x p}\right)$ positions (in $\mathrm{MeV}$ ) of the Balmer-like baryon lines together with the maximal deviation $\left(\delta^{\text {max }}\right)$ of a resonance mass from the cluster massaverage value. To keep notations transparent, the Lorentz multiplets have been represented by the quantum numbers $\{j, j\}_{B}$ of their Coulomb multiplet parts only with $B=N, \Delta$ and $\Lambda$.

\begin{tabular}{lccc}
\hline$\{\mathbf{j}, \mathbf{j}\}_{\mathbf{B}}$ & $\mathbf{M}_{\mathbf{j} \mathbf{j}}^{\text {th }}$ & $\mathbf{M}_{\mathbf{j j}}^{\text {exp }}$ & $\delta^{\text {max }}$ \\
\hline$\left\{\frac{1}{2}, \frac{1}{2}\right\}_{N}$ & 1467 & 1498 & 58 \\
$\left\{\frac{1}{2}+1, \frac{1}{2}+1\right\}_{N}$ & 1734 & 1689 & 31 \\
$\left\{\frac{1}{2}+2, \frac{1}{2}+2\right\}_{N}$ & 2068 & 2102 & 148 \\
\hline$\left\{\frac{1}{2}, \frac{1}{2}\right\}_{\Delta}$ & 1696 & 1690 & 70 \\
$\left\{\frac{1}{2}+1, \frac{1}{2}+1\right\}_{\Delta}$ & 2005 & 1922 & 28 \\
$\left\{\frac{1}{2}+2, \frac{1}{2}+2\right\}_{\Delta}$ & 2391 & 2276 & 144 \\
\hline$\left\{\frac{1}{2}, \frac{1}{2}\right\}_{\Lambda}$ & 1605 & 1508 & 103 \\
\hline
\end{tabular}

Now the apparent analogy between the spectrum of the hydrogen atom and the baryon spectra leads to the question whether or not the positions of the Lorentz clusters follow a sort of Balmer-series like pattern! The answer to that question is positive. Below quite a simple empirical recursive relation is suggested that describes with quite an amazing accuracy the reported mass averages of the resonances from the Lorentz multiplets only in terms of the cluster quantum numbers and the masses of the ground state baryons:

$$
\begin{aligned}
\frac{M_{l^{\prime}+\frac{1}{2}, l^{\prime}+\frac{1}{2}}}{M_{l^{\prime}-\frac{1}{2}, l^{\prime}-\frac{1}{2}}} & =1+\left(\frac{1}{\left(2 l^{\prime}+1\right)^{2}}-\frac{1}{\left(2 l^{\prime}+2\right)^{2}}\right) J_{l^{\prime}-\frac{1}{2}, l^{\prime}-\frac{1}{2}}^{\max }\left(J_{l^{\prime}-\frac{1}{2}, l^{\prime}-\frac{1}{2}}^{\max }+1\right), \\
J_{l^{\prime}-\frac{1}{2}, l^{\prime}-\frac{1}{2}}^{\max } & =2 l^{\prime}-\frac{1}{2}, \quad l^{\prime}>0 .
\end{aligned}
$$

Here, $\mathrm{M}_{j, j}$ denotes the mass of the respective Lorentz multiplet, while $\mathrm{J}_{j, j}^{\max }$ stands for the maximal (half-integer) spin of the multiplet $\{j, j\} \otimes\left[\left\{\frac{1}{2}, 0\right\} \oplus\left\{0, \frac{1}{2}\right\}\right]$. The

${ }^{\dagger} \mathrm{I}$ like to thank Jörg Friedrich for his remark during one of my talks that the spacing among the Höhler clusters is as well pronounced as that among the Balmer lines. 
position of the first excited nucleon cluster with $l^{\prime}=0$ is related to the nucleon mass $M_{N}$ via

$$
M_{\frac{1}{2}, \frac{1}{2}}=M_{N}+\left(1-\frac{1}{4}\right) \frac{1}{2}\left(\frac{1}{2}+1\right) M_{N}
$$

For the $\Delta$ and $\Lambda$ baryons, the mass scale entering Eq. (3), has to be replaced by $\left(M_{N}+M_{\Delta}\right) / 2$, and $\left(M_{N}+M_{\Lambda}\right) / 2$, respectively. In Table 2 , the comparison between the reported mass averages of the resonances constituting a Lorentz cluster and the positions of the predicted Balmer-like baryon lines following from Eq. (2) is given. The table leads to the insight that the pattern underlying the baryon spectrum is that of the 'Balmer-like' resonance series.

The recursive empirical mass formula in Eq. (2) may be expected to result from a proper effective quark potential having $\mathrm{O}(4)$ symmetry in the leading order. This $\mathrm{O}(4)$ symmetry has to be slightly violated if one wishes to explain the mass splitting between the members of the Lorentz clusters under consideration, that especially for the $\left\{\frac{1}{2}, \frac{1}{2}\right\}$ and $\left\{\frac{3}{2}, \frac{3}{2}\right\}$ representations turns out to be surprisingly small. Unearthing such a potential may lead to a further understanding of the nature of strong interaction and the structure of hadrons.

To conclude we wish to note that while the $\mathrm{SU}(6)_{S F}$ classification scheme for baryons may still be useful to unify the ground states of the spin- $1 / 2$ and $3 / 2$ baryons to the respective octet and decuplet, and to establish Gell-Mann's mass formulae, the relativistic $\mathrm{O}(1,3) \otimes \mathrm{SU}(2)_{I}$ symmetry is much better suited for describing the baryon excitations.

\section{Acknowledgement}

This work was supported by the Deutsche Forschungsgemeinschaft (SFB 201).

\section{References}

1. G. Höhler and H. M. Staudenmaier, in $\pi N$ Newsletter, Proceedings of the Sixth International Symposium on Meson-Nucleon Physics and the Structure of the Nucleon, ed. D. Drechsel et al. 11, 7 (1995); ibid. 194; G. Höhler, in Pion-Nucleon Scattering Landolt-Börnstein Vol. I/9b2, ed. H. Schopper (Springer, 1983).

2. B. M. K. Nefkens, ibid., p. 145.

3. M. Kirchbach, Mod. Phys. Lett. A12, 2373 (1997).

4. M. Kirchbach, Mod. Phys. Lett. A12, 3177 (1997).

5. K. Kusaka, G. Piller, A. W. Thomas, and A. G. Williams, Phys. Rev. D55, 5299 (1997).

6. Particle Data Group, R. M. Barret et al., Phys. Rev. D54, 1 (1996). 